\title{
Filtering Electrocardiogram Signals Using the Extended Kalman Filter
}

\author{
R. Sameni Student Member, IEEE, M.B Shamsollahi, Member, IEEE, C. Jutten, Member, IEEE
}

\begin{abstract}
In this paper the Extended Kalman Filter (EKF) has been used for the filtering of Electrocardiogram (ECG) signals. The method is based on a previously nonlinear dynamic model proposed for the generation of synthetic ECG signals. The results show that the EKF may be used as a powerful tool for the extraction of ECG signals from noisy measurements; which is the state of the art in applications such as the noninvasive extraction of fetal cardiac signals from maternal abdominal signals.
\end{abstract}

Index Terms-Extended Kalman Filter (EKF), Nonlinear filtering, Electrocardiography.

\section{INTRODUCTION}

$\mathrm{T}$ HE extraction of pure cardiological indices from noisy measurements, has been one of the major concerns of biomedical signal processing. Despite of the rich literature in this field, there are still many unsolved problems, which need to benefit from signal processing developments. Specifically the state of the art in applications such as fetal cardiology is to extract pure fetal ECG signals noninvasively from electrodes placed on the maternal abdomen [1]. Noting that the fetal ECG signals are very weak compared with the background noise and the maternal EMG signals, the extraction of fetal ECG signals has remained as an open problem.

On the other hand, in recent years some research has been conducted towards the generation of synthetic ECG signals. Regarding the physiological bases of ECG signals, a true ECG model should consider the morphology of the QPRST complex, together with the RR-wave timing. In a previous work [2], a synthetic model has been proposed which has unified the morphology and pulse timing of the ECG signal in a single nonlinear dynamic model. Concerning the simplicity and flexibility of this model it is believed that it can be easily adapted to a broad class of normal and abnormal ECG signals. This model may be further used in dynamic adaptive filters, such as the Kalman filter, for ECG filtering applications. The drawback of this model as a dynamical base for the Kalman filter is its nonlinearity. In

R. Sameni is a PhD candidate of Electrical Engineering in School of Electrical Engineering, Sharif University of Technology, Tehran, Iran. (Phone: +98 21661659 57; e-mail: r sameni@mehr.sharif.edu).

M. B. Shamsollahi is with the School of Electrical Engineering, Sharif University of Technology, Tehran, Iran. (e-mail: mbshams@sharif.edu).

C. Jutten is professor with Université Joseph Fourier and researcher in the Images and Signals Laboratory (CNRS UMR 5083, INPG, UJF), Grenoble, France. LIS-INPG Avenue F'elix Viallet 46, 38031 Grenoble cedex, France. (e-mail: Christian.Jutten@inpg.fr). this paper, in order to overcome the nonlinearity problem, a broader class of Kalman filters known as the Extended Kalman Filter (EKF) has been applied to normal noisy ECG data. The results show that the proposed method can fully track the ECG signal even in the epochs with a high level of noise, where the observed ECG signal is lost.

In the next section the mathematical theories of the EKF are reviewed. The third section deals with the details of the proposed method; the results of which are reported in section four. A discussion about the approach, together with some remarks on the future work has been noted in the last two sections.

\section{THEORY}

\section{A. Extended Kalman Filter Review}

A classical problem in estimation theory is the estimation of the hidden states of a system with an underlying linear or nonlinear dynamic model that is observable through a set of measurements. The Kalman filter is one of these filters and it is proved to be the optimal filter in the Minimum Mean Square Error (MMSE) sense, under some general constrains. But the conventional Kalman filter is only valid for linear models; while in practice most practical systems are nonlinear. In order to extend the Kalman filter to nonlinear systems, a modified version of it, known as the Extended Kalman Filter (EKF) has been developed [3]. Due to the issue of nonlinearity, the EKF is no longer an optimal filter in the strict sense; but in practice it has been shown that the EKF is a truly powerful adaptive filter.

For a discrete nonlinear system with the state vector $x_{k}$ and observation vector $y_{k}$, the dynamic model may be formulated as follows:

$$
\left\{\begin{array}{c}
\underline{x}_{k+1}=f\left(\underline{x}_{k}, \underline{w}_{k}, k\right) \\
\underline{y}_{k}=g\left(\underline{x}_{k}, \underline{v}_{k}, k\right),
\end{array}\right.
$$

where $\underline{w}_{k}$ and $\underline{v}_{k}$ are the process and measurement noises respectively with covariance matrices $Q_{k}=E\left\{\underline{w}_{k} \underline{w}_{k}{ }^{T}\right\}$ and $R_{k}=E\left\{\underline{v}_{k} \underline{v}_{k}^{T}\right\}$.

The initial state estimate of the state $\underline{x}_{0}$ is defined as $\underline{\bar{x}}_{0}=E\left\{\underline{x}_{0}\right\}$ with $P_{0}=E\left\{\left(\underline{x}_{0}-\underline{\bar{x}}_{0}\right)\left(\underline{x}_{0}-\underline{\bar{x}}_{0}\right)^{T}\right\}$.

In order to use a Kalman filter formalism for this system, it is necessary to derive a linear approximation of (1) near a desired reference point $\left(\underline{\hat{x}}_{k}, \underline{\hat{w}}_{k}, \underline{\hat{v}}_{k}\right)$. This approximation will lead to the following linear estimate:

$\left\{\begin{array}{c}\underline{x}_{k+1} \approx f\left(\underline{\hat{x}}_{k}, \underline{\hat{w}}_{k}, k\right)+A_{k}\left(\underline{x}_{k}-\underline{\hat{x}}_{k}\right)+F_{k}\left(\underline{w}_{k}-\underline{\hat{w}}_{k}\right) \\ \underline{y}_{k} \approx g\left(\underline{\hat{x}}_{k}, \underline{\hat{v}}_{k}, k\right)+C_{k}\left(\underline{x}_{k}-\underline{\hat{x}}_{k}\right)+G_{k}\left(\underline{v}_{k}-\underline{\hat{v}}_{k}\right)\end{array}\right.$ 
where

$$
\begin{array}{ll}
A_{k}=\left.\frac{\partial f\left(\underline{x}, \underline{\hat{w}}_{k}, k\right)}{\partial \underline{x}}\right|_{\underline{x}=\underline{\hat{x}}_{k}} & F_{k}=\left.\frac{\partial f\left(\underline{\hat{x}}_{k}, \underline{w}, k\right)}{\partial \underline{w}}\right|_{\underline{w}=\underline{\hat{w}}_{k}} \\
C_{k}=\left.\frac{\partial g\left(\underline{x}, \underline{\hat{v}}_{k}, k\right)}{\partial \underline{x}}\right|_{\underline{x}=\underline{\hat{x}}_{k}} & G_{k}=\left.\frac{\partial g\left(\underline{\hat{x}}_{k}, \underline{v}, k\right)}{\partial \underline{v}}\right|_{\underline{v}=\underline{\hat{v}}_{k}}
\end{array}
$$

In order to implement the EKF, the time propagation is done using the original nonlinear equation, while the Kalman filter gain and the covariance matrix are calculated from the linearized equations. So the EKF algorithm may be summarized as follows:

$$
\begin{gathered}
\underline{\hat{x}}_{k+1}^{-}=\left.f\left(\underline{\hat{x}}_{k}^{+}, \underline{w}, k\right)\right|_{\underline{w}=\underline{0}} \\
P_{k+1}^{-}=A_{k} P_{k}^{+} A_{k}^{T}+F_{k} Q_{k} F_{k}^{T},
\end{gathered}
$$

and the measurement propagation equations are:

$$
\begin{gathered}
\underline{\hat{x}}_{k}^{+}=\underline{\hat{x}}_{k}^{-}+K_{k}\left[\underline{y}_{k}-\left.g\left(\underline{\hat{x}}_{k}^{-}, \underline{v}, k\right)\right|_{\underline{v}=\underline{0}}\right] \\
K_{k}=P_{k}^{-} C_{k}^{T}\left[C_{k} P_{k}^{-} C_{k}^{T}+G_{k}\right]^{-1} \\
P_{k}^{+}=P_{k}^{-}-K_{k} C_{k} P_{k}^{-},
\end{gathered}
$$

where $\underline{\hat{x}}_{k}^{-}=\hat{E}\left\{\underline{x}_{k} \mid \underline{y}_{k-1}, \underline{y}_{k-2}, \ldots, \underline{y}_{1}\right\}$ is an estimate of the state vector, in the $k^{\text {th }}$ stage, using the observations $\underline{y}_{1}$ to $\underline{y}_{k-1}$, and $\underline{\hat{x}}_{k}^{+}=\hat{E}\left\{\underline{x}_{k} \mid \underline{y}_{k}, \underline{y}_{k-1}, \ldots, \underline{y}_{1}\right\}$ is an estimate of this state vector after adding the $k^{\text {th }}$ observations $\underline{y}_{k}$. $P_{k}^{-}$and $P_{k}^{+}$are defined in the same manner to be the estimates of the covariance matrices, in the $k^{\text {th }}$ stage, before and after using the $k^{\text {th }}$ observation, respectively.

As it is seen from (4) and (5), the EKF linearizes the nonlinear model in the vicinity of the previous estimated point [4], [5].

\section{B. Synthetic Electrocardiogram}

MCSharry et al. [2] have proposed a synthetic ECG generator, which is based on a nonlinear dynamic model. This model has several parameters, which makes it adaptable to many normal and abnormal ECG signals. As it may be seen in (6) the dynamic model consists of a three dimensional state equation, which generates a trajectory with the coordinate $(x, y, z)$.

$$
\begin{aligned}
& \dot{x}=\alpha x-\omega y \\
& \dot{y}=\alpha y+\omega x \\
& \dot{z}=-\sum_{i \in\{P, Q, R, S, T\}} a_{i} \Delta \theta_{i} \exp \left(-\frac{\Delta \theta_{i}^{2}}{2 b_{i}^{2}}\right)-\left(z-z_{0}\right)
\end{aligned}
$$

In this equation $\alpha=1-\sqrt{x^{2}+y^{2}}, \Delta \theta_{i}=\left(\theta-\theta_{i}\right) \bmod (2 \pi)$, $\theta=\operatorname{atan} 2(y, x)$ (the four quadrant arctangent of the real parts of the elements of $x$ and $y$, with $-\pi \leq \operatorname{atan} 2(y, x) \leq \pi$, and $\omega$ is the angular velocity of the trajectory as it moves around the limit cycle [2]. The baseline wander of the ECG signal has been modeled with $z_{0}$, which is coupled with the respiratory frequency $f_{2}$ :

$z_{0}=A \sin \left(2 \pi f_{2} t\right), \quad A=0.15 m V, \quad f_{2}=0.25 \mathrm{~Hz}$

The values of the parameters of (6) are listed in Table I.

TABLE I

PARAMETERS OF THE ECG MODEL OF (1) [2]

\begin{tabular}{cccccc}
\hline Index (i) & $\mathrm{P}$ & $\mathrm{Q}$ & $\mathrm{R}$ & $\mathrm{S}$ & $\mathrm{T}$ \\
\hline Time (Sec.) & -0.2 & -0.05 & 0 & 0.05 & 0.3 \\
$\theta_{i}$ (rads.) & $-\pi / 3$ & $-\pi / 12$ & 0 & $\pi / 12$ & $\pi / 2$ \\
$a_{i}$ & 1.2 & -5.0 & 30.0 & -7.5 & 0.75 \\
$b_{i}$ & 0.25 & 0.1 & 0.1 & 0.1 & 0.4 \\
\hline
\end{tabular}

In fact the three dimensional trajectory which is generated from (6), consists of a circular limit cycle which is pushed up and down when it approaches one of the $\mathrm{P}, \mathrm{Q}, \mathrm{R}, \mathrm{S}$ or T points. The projection of these trajectory points on the $z$ axis gives a synthetic ECG signal. The three dimensional trajectory and a typical synthetic ECG generated from the values of Table I may be seen in figs. 1 and 2.

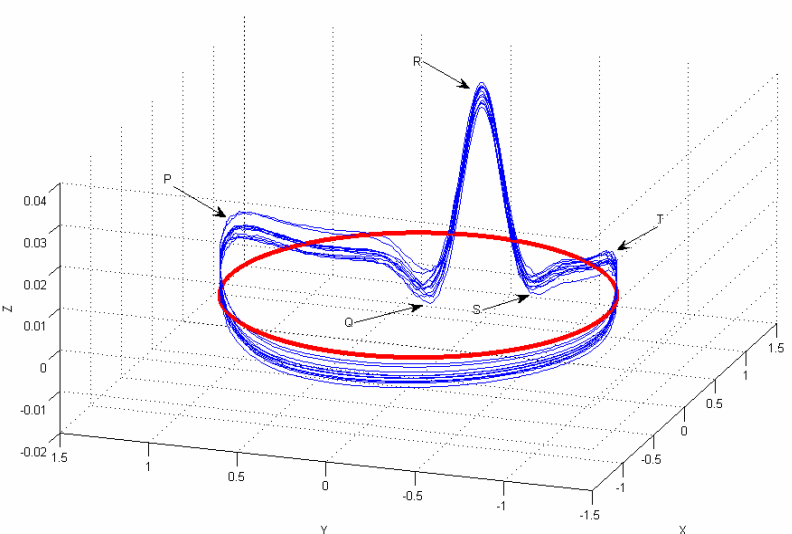

Fig. 1. The synthetic ECG trajectory generated by (6) [2]

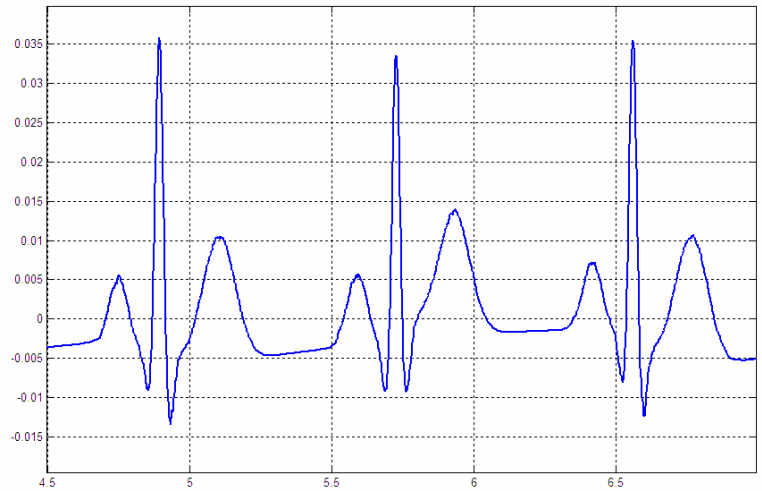

Fig. 2. A typical synthetic ECG signal generated by (6) [2]

By applying some random deviations to the parameters of Table I, it is possible to generate a rather semi-periodic signal, which is more alike to a realistic ECG signal. 


\section{METHOD}

\section{A. Linearization of the Nonlinear Dynamic ECG Model}

In order to set up an EKF model based on the nonlinear synthetic model of (6), the first step was to linearize the nonlinear model using (2) and (3). Accordingly (8) and (9) represent a linearized version of (6) with respect to the state variables $x, y$ and $z$.

$$
\begin{aligned}
& \left\{\begin{array}{l}
\dot{x}=F(x, y, z) \\
\dot{y}=G(x, y, z) \\
\dot{z}=H(x, y, z)
\end{array}\right. \\
& \frac{\partial F}{\partial x}=\frac{-2 x^{2}-y^{2}}{\sqrt{x^{2}+y^{2}}}+1 \quad \frac{\partial G}{\partial x}=\frac{-x y}{\sqrt{x^{2}+y^{2}}}+\omega \\
& \frac{\partial F}{\partial y}=\frac{-x y}{\sqrt{x^{2}+y^{2}}}-\omega \quad \frac{\partial G}{\partial y}=\frac{-2 y^{2}-x^{2}}{\sqrt{x^{2}+y^{2}}}+1 \\
& \frac{\partial H}{\partial x}=\sum_{i \in\{P, Q, R, S, T\}} \frac{a_{i} y}{x^{2}+y^{2}} \exp \left(-\frac{\Delta \theta_{i}^{2}}{2 b_{i}^{2}}\right)\left[1-\frac{\Delta \theta_{i}^{2}}{b_{i}^{2}}\right] \\
& \frac{\partial H}{\partial y}=\sum_{i \in\{P, Q, R, S, T\}} \frac{-a_{i} x}{x^{2}+y^{2}} \exp \left(-\frac{\Delta \theta_{i}^{2}}{2 b_{i}^{2}}\right)\left[1-\frac{\Delta \theta_{i}^{2}}{b_{i}^{2}}\right] \\
& \frac{\partial F}{\partial z}=0 \quad \frac{\partial H}{\partial z}=0 \quad-1
\end{aligned}
$$

Note that in order to model the effects of the mismatch of the dynamic model with a true ECG signal it is necessary to encounter a process noise in the dynamic model. For this an additive random Gaussian noise has been assumed in the dynamic model of (6). This small portion of noise, even for a truly noise-free dynamic model, gives more flexibility to the Kalman filter, and prevents it from converging to undesired limit cycles.

\section{B. Construction of the EKF Model}

Having linearized the ECG dynamic model, an EKF may be developed.

The relationship between the states and measurements of the proposed EKF depends on the location of the electrodes and the origin of the measurement noise. For example motion artifacts, environmental noises or bioelectrical artifacts such as the Electromyogram (EMG) or the Electrogastrogram (EGG), may be assumed as the measurement noises. While the measurement noise can generally take a complex and nonlinear form, the results of this paper are based on a simple additive Gaussian noise. So defining the state vector $\underline{X}_{k}=\left[\begin{array}{lll}x_{k} & y_{k} & z_{k}\end{array}\right]^{\mathrm{T}}$, the observation may be related to the state vector as follows:

$$
s_{k}=\left[\begin{array}{lll}
0 & 0 & 1
\end{array}\right] \underline{X}_{k}+v_{k}
$$

with $R_{k}=E\left\{\underline{v}_{k} \underline{v}_{k}^{T}\right\}$. Note that in the case of a single channel observation $R_{k}$ is a scalar value rather than a matrix.

In the context of estimation theory, the variance of the observation noise in (10) somehow represents the degree of reliability of a single observation. In other words, when a rather precise measurement of the states of a system is valid the value of $R_{k}$ is low, and the Kalman filter gain is adapted such as to rely on that specific measurement. While for the epochs that the measurements are too noisy or there are no measurements available, the value of $R_{k}$ is high and the Kalman filter tries to follow its underlying dynamics rather than relying on the observations. This intuition about the observation noise will be further referred to in the following section.

\section{RESULTS}

The proposed EKF model was finally implemented in Matlab ${ }^{\circledR}$ based on an approach previously reported in [5].

The ECG consisted of a normal sinus rhythm, taken from the PhysioNet ECG database [6]. The sampling frequency of the dataset was $125 \mathrm{~Hz}$. The mean ECG pulse rate was $1.2 \mathrm{~Hz}$ or 72 beats per minute (BPM), which is modeled with the $\omega$ parameter in the ECG model of (6) (divided by $2 \pi$ ); and is generally controlled by the Heart Rate Variability (HRV) of the ECG signal. A discussion about the effect of $\omega$ will be later presented.

Figs. 3 and 4 represent the ECG signal together with the

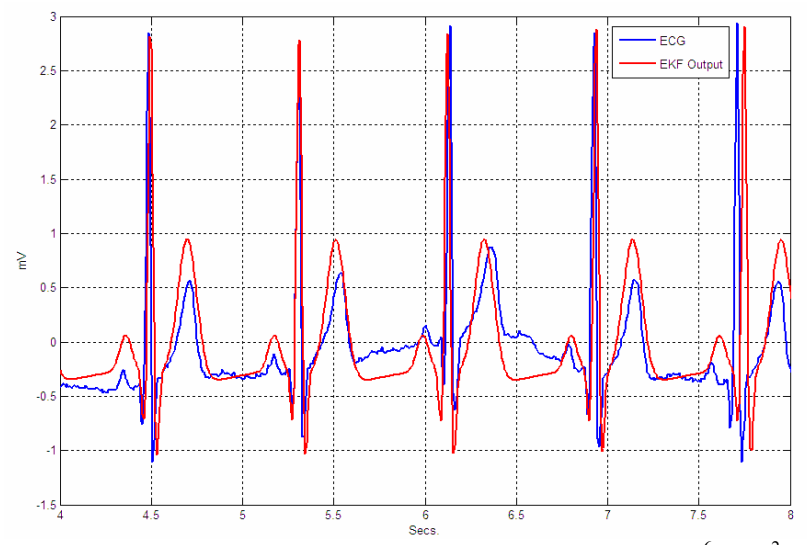

Fig. 3. The EKF output for observation variance $R_{k}=10^{6}(\mathrm{mV})^{2}$

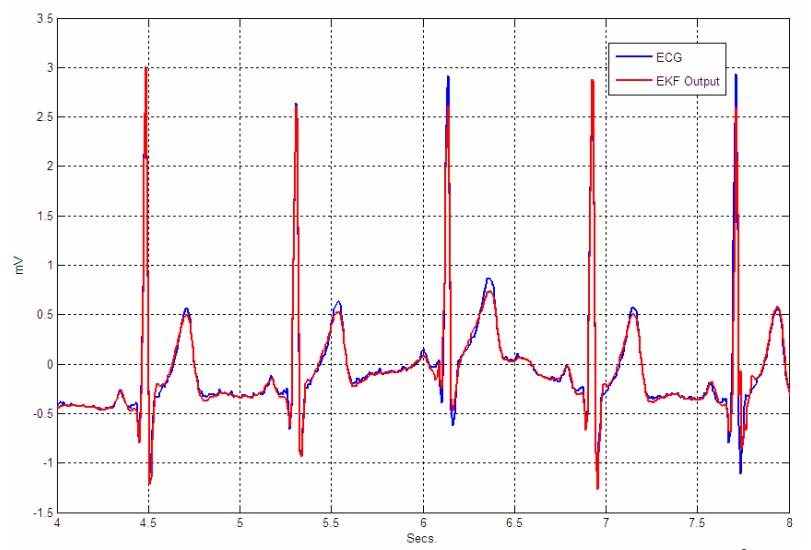

Fig. 4. EKF output for observation variance $R_{k}=1(\mathrm{mV})^{2}$

estimated ECG using the EKF, for two different values of the observation variance, namely $R_{k}=10^{6}(\mathrm{mV})^{2}$ and $R_{k}=1(\mathrm{mV})^{2}$. 
As it is seen for high values of measurement noise, the Kalman filter follows the dynamic model of (6), which generates an ECG signal similar to fig. (2). While for low values of $R_{k}$ the Kalman filter follows the measured ECG signal. In fact $R_{k}=10^{6}(\mathrm{mV})^{2}$ and $R_{k}=1(\mathrm{mV})^{2}$, may be assumed as two extreme cases for the variance of the measurement noise, while in practice the variance noise may be between these two extreme values. The results of an intermediate value are depicted in fig. 5 for a noisy segment of the ECG signal.

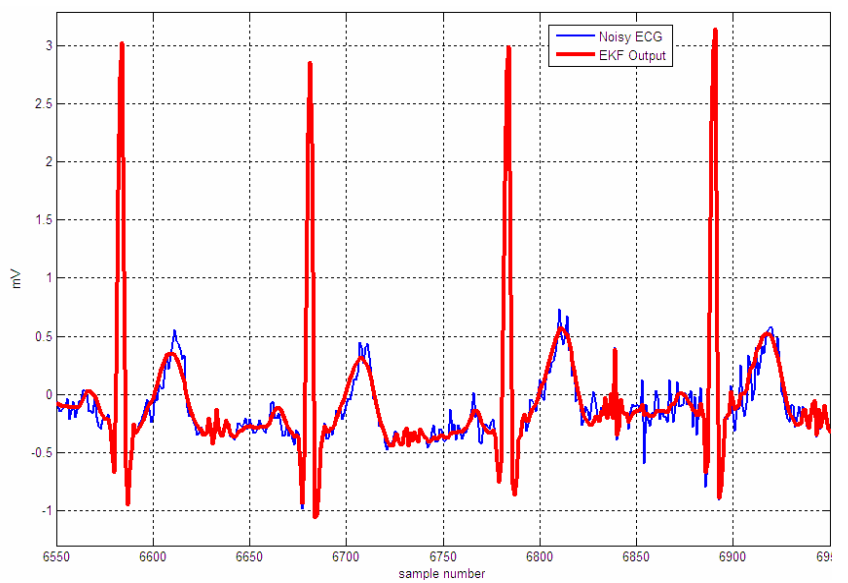

Fig. 5. EKF output for moderate measurement variance noise

In fig. 6 similar results are depicted for an ECG signal with an erased segment. In this case, before and after the erased segment, the EKF filters the ECG signal based on its internal dynamic model together with the measurements, and throughout the erased interval, the EKF works with the internal dynamic alone.

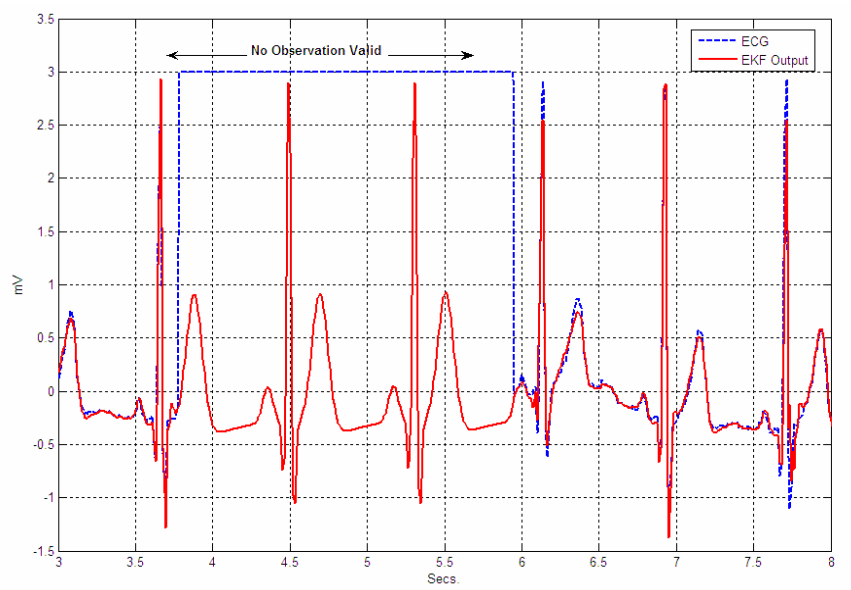

Fig. 6. EKF output for ECG signals with erased segments

\section{DISCUSSION}

In this paper an EKF was designed for the filtering of ECG signals. The EKF's dynamic model was based on a three dimensional nonlinear dynamic model previously introduced for the generation of synthetic ECG signals. This nonlinear model was linearized in order to be used in an EKF. The designed filter was later applied to normal ECG signals, and the results show the filter's capability in tracking and filtering noisy ECG signals. The proposed method can serve as a base for the design of a robust ECG filter, with vast applications for low SNR ECG signals such as the noninvasive fetal cardiac signal extraction.

In this work, the variance of the measurement noise was manually selected, while it is possible to automate this selection by estimating the noise level. It is also possible to make some deviations to the initial dynamical model proposed by McSharry et al. to encounter for the different abnormalities.

As mentioned before the HRV information may be encountered in this model via the $\omega$ parameter. There are many reported works on the spectral specifications of the HRV in the literature which may be used to modify the proposed model.

The method may be further generalized by encountering the parameters of Table I as random variables, instead of constant values, which would give a more realistic ECG signal. In this case the variance of each parameter should be estimated from typical ECG signals and the nonlinear dynamical equation should be further linearized with respect to these random variables using (2) and (3).

The evaluation of the EKF implemented in this paper was quite qualitative. In practical applications it is necessary to represent more quantitative measures, together with issues concerning the stability and convergence of the Kalman filter.

\section{CONCLUSION}

The results of this paper approve the applicability of the Extended Kalman Filter (EKF), for the filtering of noisy ECG signals. This model is highly reliant on the underlying dynamics assumed for the ECG signal. It was shown that by using a flexible nonlinear dynamical model, together with the EKF, it is possible to construct a filter which can remove environmental noises and artifacts. This filter may find vast applications for low SNR ECG signals such as the fetal ECG. Future works include the combination of the proposed EKF model with source separation techniques, for the extraction of maternal and fetal cardiac signals from multichannel surface electrode recordings.

\section{REFERENCES}

[1] F. Vrins, V. Vigneron, C. Jutten, and M. Verleysen, "Abdominal Electrodes Analysed by Statistical Processing For Fetal Electrocardiogram Extraction", Proc. of the $2^{\text {nd }}$ Inter. Conf. on Biomed. Eng. Feb. 16-18, 2004, Innsbruck, Austria, pp. 244-249.

[2] P. E. McSharry, G. D. Clifford, L. Tarassenko, L. A Smith, "A Dynamic Model for Generating Synthetic Electrocardiogram Signals", IEEE Trans. Biomed. Eng., vol. 50, No. 3, Mar. 2003, pp. 289-294.

[3] S. M. Kay, Fundamentals of Statistical Signal Processing: Estimation Theory, Prentice Hall PTR, 1993.

[4] M. S. Grewal, A. P. Andrews, Kalman Filtering: Theory and Practice Using Matlab ${ }^{\circledR}, 2$ nd Edition, John Wiley \& Sons Inc., 2001.

[5] M. Nørgaard, "The Kalman Filter Toolbox", Online Available: http://www.iau.dtu.dk/research/control/kalmtool.html

[6] PhysioNet, http://www.physionet.org/physiobank/database/nsrdb/ 